\title{
Broadening Genetic Diversity of the Japanese Potato Gene Pool
}

\author{
Kazuyoshi Hosaka ${ }^{1}$ (D) $\cdot$ Rena Sanetomo ${ }^{1}$
}

Published online: 29 January 2020

(C) The Author(s) 2020

\begin{abstract}
Newly developed potato varieties in Japan have never replaced existing old varieties, indicating genetic poverty in our gene pool. To broaden the genetic diversity, Andean primitive cultivated potatoes (Andigena) were used to create parental clones. A total of 184 Andigena accessions and 10 tetraploid Solanum tarijense-Andigena hybrid clones were crossed with a breeding clone 10H17. From $6939 \mathrm{~F}_{1}$ hybrid clones, 287 were selected as long-day-adapted $F_{1}$ clones. From progeny evaluation using these as pollen parents crossed with Atlantic, 95 male-fertile and high-yielding $\mathrm{F}_{1}$ clones were selected (PGEL clones). The genetic relationship was evaluated using 17,947 single nucleotide polymorphism (SNP) markers, which disclosed their distinctiveness, with larger mean Euclidean distances between PGEL clones and Japanese, foreign, or all tetraploid varieties (48.0, 49.9 or 49.4, respectively) than between Japanese and foreign varieties (47.2). Thus, these PGEL clones can be used to expand genetic diversity in Japanese potato breeding populations.
\end{abstract}

\section{Resumen}

Las nuevas variedades de papa desarrolladas en Japón nunca han reemplazado a las viejas variedades en existencia, indicando pobreza genética en nuestro grupo de genes. Para ampliar la diversidad genética, se usaron papas cultivadas primitivas Andinas (Andigena) para crear clones parentales. Se cruzaron un total de 184 accesiones de Andigena y 10 clones híbridos tetraploides de Solanum tarijense-Andigena, con el clon 10H17. De 6939 clones híbridos de F1, se seleccionaron 287 como clones F1 adaptados a día largo. De la evaluación de la progenie usando polen parental cruzado con Atlantic, se seleccionaron 95 clones androfértiles y de alto rendimiento de F1 (clones PGEL). Se evaluó la relación genética usando 17,947 marcadores de polimorfismo de nucleótido simple (SNP), que exhibieron sus diferencias, con promedios de distancias Euclideanas mas grandes entre los clones PGEL y las variedades japonesas, extranjeras, o todas las tetraploides $(48.0,49.9$ o 49.4, respectivamente) que entre variedades japonesas y extranjeras (47.2). De aqui que estos clones PGEL pueden usarse para expandir la diversidad genética en las poblaciones del mejoramiento genético de papa japonés.

Keywords Andigena Long-day-adapted $\mathrm{F}_{1}$ hybrid clones PGEL clones $\cdot$ Single nucleotide polymorphism (SNP) Marker-assisted selection · Cytoplasmic genome diversity

This paper is dedicated to Mr. Masahiko Matsuo, the former President of Calbee Inc., who endowed the Potato Germplasm Enhancement Laboratory at Obihiro University of Agriculture and Veterinary Medicine to initiate the prebreeding program presented in this paper but suddenly died at the age of 76 on February 12, 2018.

Electronic supplementary material The online version of this article (https://doi.org/10.1007/s12230-020-09762-8) contains supplementary material, which is available to authorized users.

Kazuyoshi Hosaka spudman@obihiro.ac.jp

1 Potato Germplasm Enhancement Laboratory, Obihiro University of Agriculture and Veterinary Medicine, Obihiro, Hokkaido 080-8555, Japan

\section{Introduction}

Genetic variation is necessary for crop improvement. The larger the genetic diversity in the breeder's gene pool, the greater the potential genetic gain (Kawano 2003). Potato (Solanum tuberosum L.) suffered severe damage by late blight, caused by Phytophthora infestans, in the 1840s, which significantly reduced the genetic diversity which was present among the first introductions into Europe in the early sixteenth century (Provan et al. 1999). A Chilean potato, Rough Purple Chili, was introduced by C. Goodrich in 1851, which substantially initiated modern potato breeding. Early Rose, a grandchild of Rough Purple Chili, produced many descendant varieties worldwide (Plaisted and Hoopes 1989), which resulted 
in the narrow genetic base of modern potato varieties (Mendoza and Haynes 1974).

During the past several decades, general usage trends from table to processing potatoes have caused significant genetic differentiation. For instance, North American varieties were differentiated into four distinct groups: the chip processing group, the French fry processing and table russet group, the yellow and round white table group, and the pigmented group (Hamilton et al. 2011; Hirsch et al. 2013). In Europe, three divergent groups, consisting of heirloom cultivars, frying cultivars, and cultivars used for starch processing, were identified (Uitdewilligen et al. 2013). These genetic differentiations were always accomplished by the use of Andean landraces and their closely related wild species (Ross 1986; Plaisted and Hoopes 1989; Vos et al. 2015).

New sources of genetic variation have been introduced in recent decades. Late blight resistance has been incorporated from $S$. demissum since the beginning of the twentieth century (Ross 1986). Resistance to Potato virus $Y$ was incorporated from S. stoloniferum, S. tuberosum ssp. andigena, and S. chacoense (Munoz et al. 1975; Asama et al. 1982; Ross 1986; Song and Schwarzfischer 2008). Resistances to golden cyst nematode (Globodera rostochiensis) and white cyst nematode (G. pallida) were incorporated from $S$. tuberosum ssp. andigena, S. spegazzinii, and S. vernei (Huijsman 1955; Ross and Huijsmann 1969). Accumulation of reducing sugar in tubers after cold storage (cold-sweetening) results in undesirably dark color chips or fries. Cold-sweetening resistance was incorporated from S. chacoense (Akeley et al. 1968), S. phureja (Ehlenfeldt et al. 1990), and S. tarijense (Hermundstad 1986), which was a driving force of differentiation into three distinct groups of chip processing varieties in North America (Hirsch et al. 2013). Nevertheless, the majority of genetic variation that was present 100 years ago is still present in modern cultivars, and an increase in genetic variation in the potato gene pool has occurred with the introduction of these new Solanum species (Vos et al. 2015), although genetic improvement for total tuber yield has not been realized in the USA over the last century (Douches et al. 1996).

In Japan, two old introduced varieties, Irish Cobbler (a US variety first reported in 1876) and May Queen (a UK variety raised in 1900), are still leading varieties for table use. Toyoshiro (Sakaguchi et al. 1976) and Konafubuki (Asama et al. 1982) are chip processing and starch processing varieties, respectively. These four major varieties are not resistant to golden cyst nematodes, which seriously threaten presentday potato production in Japan (Mori et al. 2007). Although many resistant varieties were released in the past four decades, Irish Cobbler, May Queen, Toyoshiro, and Konafubuki are still major varieties. Igarashi et al. (2019) evaluated the genetic diversity of Japanese potatoes using a single nucleotide polymorphism (SNP) array. Recently released chip processing varieties were genetically differentiated from other Japanese potatoes because North American chip processing varieties contributed greatly to the Japanese ones (Igarashi et al. 2019). However, other Japanese potatoes, including those for starch processing, were not clearly differentiated to specific market types. Only double-cropping varieties formed a unique group because strong selection pressure was applied to the cultivation system (spring-season product is used as seed for fall-season cropping). The interpopulation genetic distances between Japanese and foreign genotypes were significantly lower than the within-population distances of foreign genotypes, indicating that Japanese potatoes are not uniquely differentiated but are included within the genetic diversity of foreign genotypes (Igarashi et al. 2019). Thus, the current Japanese gene pool might be insufficient to create superior varieties due to genetic poverty.

Based on this common recognition among potato industry personnel, growers, and scientists, we launched a parental breeding (or prebreeding) program at the Potato Germplasm Enhancement Laboratory of the Obihiro University of Agriculture and Veterinary Medicine in April 2013. Our strategy to broaden genetic diversity is to use Andean native tetraploid varieties (S. tuberosum ssp. andigena; hereafter, referred to as Andigena). Andigena is widely grown from Mexico to Chile, mainly in the central Andean highlands (Hawkes 1990), and has untapped, large genetic variation (Simmonds 1964; Hosaka and Hanneman 1988; Grun 1990), making it a popular source for broadening the genetic diversity of potatoes (Glendinning 1969; Cubillos and Plaisted 1976; Tarn and Tai 1977). However, Andigena is poorly adapted to long day-length, as it matures late and yields less with numerous small, often poor-shaped, tubers when grown in the summer of northern temperate regions (Glendinning 1975a; Huarte and Plaisted 1984). We made $\mathrm{F}_{1}$ hybrids between Andigena and a male and female fertile breeding clone, $10 \mathrm{H} 17$, and selected long-day-adapted or high-tuber-yielding genotypes under our environment. This might be a similar idea to the well-known "Neo-Tuberosum" project, which was a long-term experiment designed to recreate $S$. tuberosum by recurrent mass-selection from Andigena started in 1959 in the UK (Simmonds 1966). Long-day adapted genotypes, or those producing tubers under long days, were selected and named "Neo-Tuberosum" (Simmonds 1969 in Glendinning 1975a). Independently in the USA, six cycles of phenotypic recurrent selection for yield were applied to a collection of Andigena, which resulted in increased photoperiodic adaptation and earliness (Rasco et al. 1980; Munoz and Plaisted 1981). Our approach, however, was considerably different from the "Neo-Tuberosum" experiment because we systematically used Andigena germplasm in single hybridization events, from which long-day adapted $F_{1}$ hybrids were selected and immediately released to breeders. We were particularly concerned about the cytoplasmic genome type. Cytoplasmic genomes of the cultivated potatoes have been 
distinguished into six distinct types $(\mathrm{M}, \mathrm{P}, \mathrm{A}, \mathrm{W}, \mathrm{T}$, and $\mathrm{D}$ types) (Hosaka and Sanetomo 2012). Classical European and American varieties have exclusively T-type cytoplasm, which is sensitive to nuclear genes causing male sterility (Grun et al. 1977). The cytoplasmic genome derived from S. stoloniferum (W/ $\gamma$ type) or S. demissum (D type) is also associated with male sterility (Dionne 1961; Lössl et al. 2000). As resistance breeding proceeds, the frequency of male sterility-causing cytoplasm is increasing in the breeder's gene pool, which has caused a serious problem because available pollen parents have been gradually limited (Mihovilovich et al. 2015). Thus, we avoided the use of genotypes with male sterility-causing cytoplasm and selected male fertile clones. The gain of genetic diversity by the new input of Andigena germplasm was measured using SNP markers. Breeding gain was not evaluated in this study because breeders are using the long-day adapted $F_{1}$ hybrids as parents and evaluating them in practical breeding programs.

\section{Materials and Methods}

\section{Plant Materials}

As source materials, 184 Andigena accessions were obtained as seeds from the Potato Introduction Station (NRSP-6), Sturgeon Bay, Wisconsin, USA. Ten $4 x$ taradg hybrids obtained from crosses of $S$. tarijense used as female parents and Andigena as male parents (Hosaka et al. 2018) were also used, which have T-type cytoplasm derived from $S$. tarijense but are male and female fertile. These were crossed with a breeding clone, $10 \mathrm{H} 17$, which was derived from a cross of Saikai 35 (Mori et al. 2012) as its female parent with a US variety Pike (Plaisted et al. 1998) as its male parent, and was a sister clone of Harimaru (Fujimatsu et al. 2018), both having S. phureja-derived P-type cytoplasm and good male and female fertilities. In addition, $10 \mathrm{H} 17$ has resistance genes to cyst nematode (H1), Potato virus $X(R \times 1)$, Potato virus $Y\left(R y_{c h c}\right)$, and late blight $(R 1)$. For evaluation of Andigena-10H17 hybrids, a variety known worldwide, Atlantic, was crossed.

For assessment of genetic diversity, nine major Japanese varieties (Hokkaikogane, Kitahime, Konafubuki, Nishiyutaka, Norin 1, Saya-akane, Sayaka, Toyoshiro, and Waseshiro), one Japanese landrace (Nemuromurasaki), one Japanese breeding clone (10H17), eight North American varieties (Andover, Atlantic, Early Rose, Kennebec, Norking Russet, Pike, Shepody, and Waneta), six European varieties (Alwara, Bintje, Innovator, Lady Joe, Olympus, and Sarpo Mira), and DM 1-3 516R44 (a clone used for a potato reference genome) were used to obtain $22 \mathrm{~K} \mathrm{SNP}$ data.

\section{Breeding Procedures}

Basically, the following procedures were applied: first, longday-adapted, or high-tuber-yielding Andigena genotypes were selected under natural day-length in the summer (step 1). The long-day-adapted Andigena genotypes were reciprocally crossed with $10 \mathrm{H} 17$ (step 2). The seedling progenies of $\mathrm{F}_{1}$ (Andigena $\times 10 \mathrm{H} 17$ ) hybrids were grown under natural daylength in the summer, and long-day-adapted $\mathrm{F}_{1}$ hybrids were selected (step 3). To evaluate the selected $F_{1}$ hybrids for use as parents by breeders, these were crossed as pollen parents with Atlantic (step 4), and their seedling progenies were grown under natural day-length in the summer. High-yielding families were identified, and their parental $\mathrm{F}_{1}$ hybrids were regarded as superior long-day-adapted $\mathrm{F}_{1}$ hybrids (step 5). These superior genotypes were named PGEL clones and distributed to breeders.

\section{Crossing}

Plants were grown in a pollinator-free greenhouse under $18 \mathrm{~h}$ day-length with supplementary lights during winter. Anthers and petals were removed from flower buds one or two days prior to opening, and freshly collected pollen was applied immediately to the stigmas. Berries were collected one month after pollination. After another one month of maturation, seeds were collected, dried and stored at $4{ }^{\circ} \mathrm{C}$ until use.

\section{Seedling Evaluation}

Seedling evaluations were conducted from spring to summer seasons; outside at Hokkaido Agricultural Research Center (E143 ${ }^{\circ} 04^{\prime} 34^{\prime \prime}, \mathrm{N} 42^{\circ} 53^{\prime} 25^{\prime \prime}$ ) in 2012, in a plastic house at Calbee Potato Inc. (E143 $05^{\prime} 10^{\prime \prime}$, N42 $\left.54^{\prime} 35^{\prime \prime}\right)$ in 2013, and in a greenhouse at Obihiro University of Agriculture and Veterinary Medicine (E143 ${ }^{\circ} 10^{\prime} 2^{\prime \prime}$, N42 $\left.52^{\prime} 17^{\prime \prime}\right)$ from 2014 to 2017. All windows of the greenhouse were kept open and covered with $0.3 \mathrm{~mm}$ nylon mesh.

Seeds were soaked in $2000 \mathrm{ppm}$ gibberellic acid $\left(\mathrm{GA}_{3}\right)$ solution for $48 \mathrm{~h}$, rinsed in tap water, sown in a cell tray filled with potting soil, and covered with vermiculite. After the second leaves were well expanded (usually 3 weeks after seedsowing), the young seedlings were transplanted to black vinyl pots (10.5 cm diameter) and further grown following the routine practiced in our laboratory. Plants were harvested individually. Familial means were used to evaluate seedling populations as practiced by Bradshaw and Mackay (1994). A familial mean of tuber yield ( $\mathrm{g} / \mathrm{plant}$ ) was obtained by the total tuber weight of each family divided by the number of seedlings in the family. The largest tuber from each seedling was collected, and the familial bulk was used to measure the specific gravity, calculated by aerial weight/(aerial weight - underwater weight). 


\section{Breaking Tuber Dormancy}

In order to use harvested tubers immediately for crossing in the winter, tuber dormancy was broken by a gibberellic acid treatment (Jansky and Hamernik 2015). A part of the tuber surface was removed by a knife and dipped briefly into $50 \mathrm{ppm}$ gibberellic acid $\left(\mathrm{GA}_{3}\right)$ solution. The treated tubers were placed on soil with the cut surface upside and dried for healing three days. Then, the treated tubers were covered with soil and watered.

\section{Marker Assay}

PGEL clones were characterized using molecular markers. Total DNA was extracted from fresh leaves by the method described in Hosaka and Hanneman (1998). A multiplex PCR method developed by Mori et al. (2011) was used to detect resistance genes to cyst nematode (H1), Potato virus $X(R x 1)$, Potato virus $Y\left(R y_{c h c}\right)$, and late blight ( $R 1$ and $\left.R 2\right)$. Note that for this method, Ohbayashi et al. (2010) originally developed the marker band of $R 2$. However, Iketani et al. (2015) suggested that this marker band is not linked with $R 2$ but with the field resistance QTL reported by LeonardsSchippers et al. (1994). The PCR marker included in the multiplex PCR method for $R x l$ was developed by Ohbayashi et al. (2010), which amplifies from Exon 1 to a spacer between Exon 1 and Exon 2 of the $R x 1$ sequence (Bendahmane et al. 1999). New markers 1Rx1 (annealing to Exon 1) and 5Rx1 (annealing to the promotor region), developed by Ahmadvand et al. (2013), were also used as follows: the PCR was performed in a volume of $10 \mu \mathrm{l}$ consisting of $2 \mu \mathrm{l}$ of template DNA (approximately $5 \mathrm{ng} / \mu \mathrm{l}$ ), $5 \mu \mathrm{l}$ of Ampdirect ${ }^{\circledR}$ Plus (Shimadzu Co., Japan), 0.25 units of Taq DNA polymerase (BIOTAQ ${ }^{\mathrm{TM}}$ HS DNA Polymerase, Bioline Ltd., UK), and $1 \mu \mathrm{l}$ each of $3 \mu \mathrm{M}$ forward and reverse primers. The reaction was performed using a thermal cycler (Veriti, Applied Biosystems) with a thermal profile of one cycle of $10 \mathrm{~min}$ at $95^{\circ} \mathrm{C}$, followed by 35 cycles of $30 \mathrm{~s}$ at $95^{\circ} \mathrm{C}, 30 \mathrm{~s}$ at $60^{\circ} \mathrm{C}$ for $1 \mathrm{Rx} 1$ (or $62^{\circ} \mathrm{C}$ for $5 \mathrm{Rx} 1$ ), and $1 \mathrm{~min}$ at $72{ }^{\circ} \mathrm{C}$, and a final cycle of $10 \mathrm{~min}$ at $72{ }^{\circ} \mathrm{C}$. The cytoplasm types were determined by the method described in Hosaka and Sanetomo (2012).

\section{SNP Data}

A total of 1-2 micrograms of dried DNA was sent to GeneSeek (Neogen Corporation, NE, USA) to obtain $22 \mathrm{~K}$ potato V3 SNP array data. Using a diploid genotype model, $\mathrm{AA}, \mathrm{AB}$, and $\mathrm{BB}$ were converted to the ordinal variables 1,2 , and 3 , respectively. Hierarchical cluster analysis was performed by the unweighted pair-group method with arithmetic mean (UPGMA) using the software JMP Pro 14.0.0 (SAS Institute Inc.). The $8 \mathrm{~K} \mathrm{SNP}$ array data for 247 diverse collections, consisting mostly of North American varieties and breeding clones, 25 genetic stocks, and 12 wild species, were obtained from the literature (Hirsch et al. 2013). The $8 \mathrm{~K}$ SNP data for almost all Japanese varieties were also available in Igarashi et al. (2019). As all informative SNP loci of the $8 \mathrm{~K}$ SNP array were included among the $22 \mathrm{~K}$ SNP locus data, PGEL clones were further evaluated with these diverse collections using the $8 \mathrm{~K} \mathrm{SNP}$ data. To evaluate the extent of within- and between-population differences, genotype data 1 , 2 , and 3 were treated as categorical variables, and the dissimilarities between genotypes were calculated as Euclidean distances. An overall mean of pairwise Euclidean distances was obtained for both within and between populations.

\section{Results}

\section{Breeding Process}

Four selection cycles were conducted following the basic procedures with slight modifications in each selection cycle (Fig. 1). The selection process in each selection cycle is described in detail in Appendix.

Most Andigena accessions used in the first selection cycle were used again in either the second or the third selection cycle. Thus, a total of 2190 genotypes of 184 Andigena accessions (1-15 seedlings per accession) were grown, from which 325 were selected for crossing with $10 \mathrm{H} 17$. In the fourth selection cycle, 10 clones of $4 x$ tar-adg hybrids were crossed with $10 \mathrm{H} 17$. A total of $6939 \mathrm{~F}_{1}$ hybrid plants with $10 \mathrm{H} 17$ were created, from which 287 were selected as longday-adapted $F_{1}$ hybrid clones. Based on our evaluation of 6949 plants of Atlantic $\times$ long-day-adapted $\mathrm{F}_{1}$ hybrid clones, 95 superior parents were selected as PGEL clones and released in six years (Table 1). These 95 PGEL clones were descended from 49 Andigena accessions and four clones of $4 x$ tar-adg hybrids.

\section{Tuber Yields in Andigena}

Andigena accessions evaluated for the third selection cycle in 2014 consisted of 41 Argentine, 24 Bolivian and one each of Mexican and Colombian accessions, which were grown in a greenhouse. The mean tuber yield obtained from seedlings of Bolivian accessions was $13.9(\mathrm{SD}=8.54) \mathrm{g} / \mathrm{plant}$, which was not significantly different from that of the Argentine accessions ( $14.4 \mathrm{~g} /$ plant, $\mathrm{SD}=7.92)$. In contrast, Andigena accessions evaluated for the second selection cycle were grown outside under harsh conditions (sometimes very windy). These were collections from a broader range from Mexico to Chile (Fig. 2). One-way ANOVA indicated a significant difference between countries of origin $(P<0.0001)$. As shown in Fig. 2, tuber yields increased as the country of origin went 


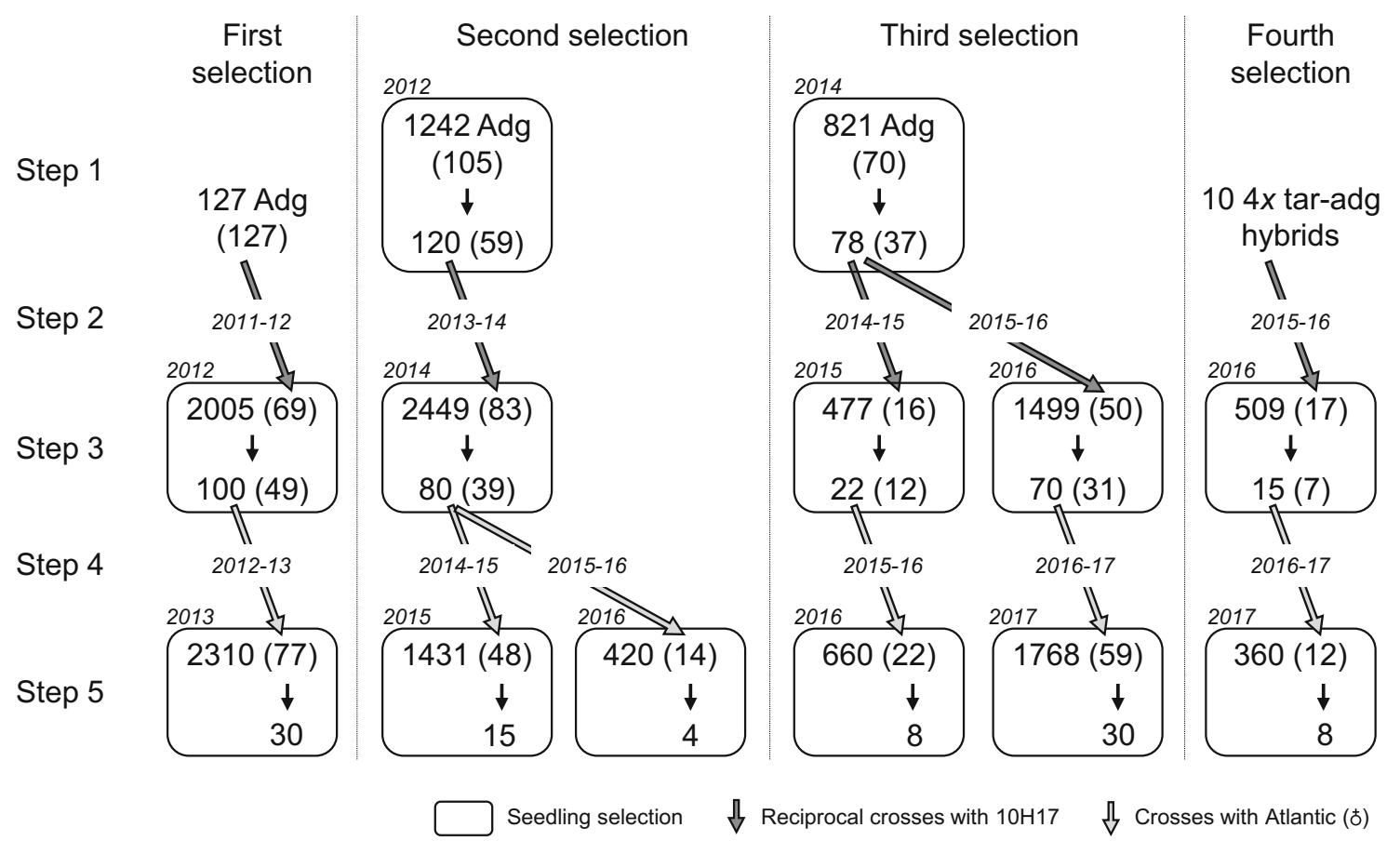

Fig. 1 Breeding processes to create superior long-day-adapted clones (PGEL clones). The number of genotypes and families in parentheses are represented

farther from the equatorial country (Ecuador) toward the southern and northern edges of the cultivation areas (Supplementary Table 1). However, as also shown in Fig. 2, 49 Andigena accessions that finally contributed to PGEL clones were not always high-yielding accessions. For example, 14 high-yielding genotypes from Chilean Andigena accessions were selected for crossing with $10 \mathrm{H} 17$. However, some emerged late, some were male sterile, and some setting berries were damaged by a freezing accident (see Appendix). As a result, only one was successfully crossed with Atlantic, but its progeny did not yield well. Therefore, the tuberyielding ability of PGEL clones was not always associated with the place of origin of the Andigena accessions.

\section{Yield Levels among Breeding Steps}

Because one selection cycle was completed in 3 to 4 years and the three populations at different improvement steps [Andigena, $\mathrm{F}_{1}$ (Andigena $\times 10 \mathrm{H} 17$ ), and Atlantic $\times \mathrm{F}_{1}$ hybrid populations] were evaluated at seedling stages, it was impossible to compare the three populations, derived from a set of Andigena accessions, simultaneously in the same year. Furthermore, the growing conditions of seedlings differed considerably by year (see Materials and methods). Thus, the populations grown in the same year, but of different selection cycles, were compared for tuber yield (Table 2). Four sets of populations at different improvement steps were grown in different years. In all comparisons, the population means were significantly higher in the $F_{1}$ (Andigena $\times 10 \mathrm{H} 17$ ) population than in the Andigena population and highest in the Atlantic $\times$ $\mathrm{F}_{1}$ hybrid population (Table 2).

\section{Characterization of PGEL Clones}

The parental clone $10 \mathrm{H} 17$ has $H 1, R 1, R x 1$, and $R y_{\text {chc }}$ genes. Marker analysis using the multiplex PCR method revealed that out of 95 PGEL clones, 77, 81, 70, and 44 had H1, R1, $R x 1$, and $R y_{c h c}$, respectively (Fig. 3, Table 1). Twenty-three PGEL clones had all marker bands for the four resistance genes. The presence/absence of the $1 \mathrm{Rx} 1$ marker band was perfectly matched with the Rx1 marker of the multiplex PCR. However, 12 positive PGEL clones lacked the 5Rx1 marker band (Table 1).

The transmission rate from $10 \mathrm{H} 17$ to PGEL clones was examined by chi-square test. The transmission rate of $46.3 \%$ for $R y_{c h c}$ indicates that the cross was $10 \mathrm{H} 17$ (simplex) $\times$ Andigena (nulliplex). The other three genes were significantly overrepresented (Supplementary Table 2). The best fit for $\mathrm{HI}$ and $R 1$ were duplex in $10 \mathrm{H} 17$ and nulliplex in Andigena. For $R x 1$, simplex $\times$ simplex was the best fit. However, for the $5 \mathrm{Rx} 1$ marker the possibility that $10 \mathrm{H} 17$ was simplex and Andigena nulliplex could not be excluded.

Cytoplasm types were determined (Table 1). PGEL clones with $10 \mathrm{H} 17$ as female parents had P-type cytoplasm (62 clones). Another one clone had P-type cytoplasm, which was likely derived from the Andigena female. A-, M-, and W-type cytoplasms were found in 20,3, and 3 PGEL clones, respectively. Six PGEL clones had T-type cytoplasm, which 
Table 1 Characterization of 95 long-day-adapted hybrid clones (PGEL clones). The presence (+) or absence (-) of resistance genes were estimated by marker analysis

\begin{tabular}{|c|c|c|c|c|c|c|c|c|}
\hline Clone & Cytoplasm & Pedigree (female $\times$ male); accession and origin of the Andigena parent & $H 1$ & $R 1$ & $R x 1$ & $1 \mathrm{Rx}^{1)}$ & $5 \mathrm{R} \times 1^{1)}$ & $R y_{c h c}$ \\
\hline $12 \mathrm{H} 143-1 \mathrm{H}$ & $\mathrm{P}$ & $10 \mathrm{H} 17 \times 2$ S15-2 (adg PI 214436; Junin, Peru) & + & + & + & + & + & + \\
\hline $12 \mathrm{H} 149-1$ & $\mathrm{P}$ & 10H17 × 2S59-1 (adg PI 246545; Cuzco, Peru) & - & + & + & + & + & - \\
\hline $12 \mathrm{H} 151-2$ & $\mathrm{P}$ & 10H17 × 2S79-2 (adg PI 280990; La Paz, Bolivia) & - & + & + & + & + & + \\
\hline $12 \mathrm{H} 153-1$ & $\mathrm{P}$ & $10 \mathrm{H} 17 \times 2$ S2-2 (adg PI 161683; Tlaxcala, Mexico) & - & + & - & - & - & - \\
\hline $12 \mathrm{H} 155-1 \mathrm{~S}$ & $\mathrm{P}$ & $10 \mathrm{H} 17 \times 2$ S97-2 (adg PI 281064; Cuzco, Peru) & + & + & - & - & - & - \\
\hline $12 \mathrm{H} 164-1$ & $\mathrm{P}$ & $10 \mathrm{H} 17 \times 2$ S142-1 (adg PI 473277; Jujuy, Argentina) & + & + & + & + & + & + \\
\hline $12 \mathrm{H} 165-1$ & $\mathrm{P}$ & $10 \mathrm{H} 17 \times 2$ S147-2 (adg PI 473284; Salta, Argentina) & + & + & + & + & + & + \\
\hline $12 \mathrm{H} 165-2$ & $\mathrm{P}$ & $10 \mathrm{H} 17 \times 2$ S147-2 (adg PI 473284; Salta, Argentina) & + & + & + & + & + & + \\
\hline $12 \mathrm{H} 165-3 \mathrm{H}$ & $\mathrm{P}$ & $10 \mathrm{H} 17 \times 2$ S147-2 (adg PI 473284; Salta, Argentina) & + & + & - & - & - & + \\
\hline $12 \mathrm{H} 168-1$ & $\mathrm{P}$ & $10 \mathrm{H} 17 \times 2$ S158-1 (adg PI 473296; Jujuy, Argentina) & + & + & + & + & + & - \\
\hline $12 \mathrm{H} 173-1 \mathrm{H}$ & $\mathrm{P}$ & $10 \mathrm{H} 17 \times 2$ S175-1 (adg PI 498310; La Paz, Bolivia) & + & + & - & - & - & - \\
\hline $12 \mathrm{H} 176-2$ & $\mathrm{P}$ & $10 \mathrm{H} 17 \times 2$ S191-1 (adg PI 558137; Salta, Argentina) & + & + & + & + & + & - \\
\hline $12 \mathrm{H} 178-2$ & $\mathrm{P}$ & 10H17 × 2S198-2 (adg PI 473393; Potosi, Bolivia) & - & + & + & + & + & + \\
\hline $12 \mathrm{H} 180-1$ & A & 2S1-1 (adg PI 161350; Puebla, Mexico) × 10H17 & + & + & + & + & + & + \\
\hline $12 \mathrm{H} 184-1$ & A & 2S47-1 (adg PI 243430; Tolima, Colombia) $\times 10 \mathrm{H} 17$ & + & + & - & - & - & - \\
\hline $12 \mathrm{H} 184-2$ & A & 2S47-1 (adg PI 243430; Tolima, Colombia) $\times 10 \mathrm{H} 17$ & + & - & - & - & - & - \\
\hline $12 \mathrm{H} 185-2$ & A & 2S49-1 (adg PI 243434; Santander, Colombia) × 10H17 & + & - & + & + & + & - \\
\hline $12 \mathrm{H} 187-1$ & A & 2S59-1 (adg PI 246545; Cuzco, Peru) $\times 10 \mathrm{H} 17$ & + & + & + & + & + & + \\
\hline $12 \mathrm{H} 187-2$ & A & 2S59-1 (adg PI 246545; Cuzco, Peru) $\times 10 \mathrm{H} 17$ & + & + & + & + & + & - \\
\hline $12 \mathrm{H} 188-2$ & W & 2S65-2 (adg PI 255508; Chuquisaca, Bolivia) × 10H17 & + & + & - & - & - & - \\
\hline $12 \mathrm{H} 189-2$ & $\mathrm{M}$ & 2S73-1 (adg PI 258936; La Paz, Bolivia) × 10H17 & + & + & + & + & + & + \\
\hline $12 \mathrm{H} 196-2$ & $\mathrm{P}$ & 2S133-1 (adg PI 473261; Salta, Argentina) $\times 10 \mathrm{H} 17$ & - & + & + & + & + & - \\
\hline $12 \mathrm{H} 197-1 \mathrm{H}$ & A & 2S138-1 (adg PI 473269; Salta, Argentina) $\times 10 \mathrm{H} 17$ & + & + & + & + & + & + \\
\hline $12 \mathrm{H} 198-1$ & A & 2S139-2 (adg PI 473270; Salta, Argentina) $\times 10 \mathrm{H} 17$ & + & + & + & + & + & - \\
\hline $12 \mathrm{H} 198-2$ & A & 2S139-2 (adg PI 473270; Salta, Argentina) $\times 10 \mathrm{H} 17$ & + & - & + & + & + & - \\
\hline $12 \mathrm{H} 200-1$ & A & 2S158-1 (adg PI 473296; Jujuy, Argentina) $\times 10 \mathrm{H} 17$ & + & + & - & - & - & + \\
\hline $12 \mathrm{H} 201-1$ & A & 2S161-1 (adg PI 473300; Salta, Argentina) $\times 10 \mathrm{H} 17$ & + & + & + & + & + & + \\
\hline $12 \mathrm{H} 201-2$ & A & 2S161-1 (adg PI 473300; Salta, Argentina) × 10H17 & + & + & + & + & + & - \\
\hline $12 \mathrm{H} 206-1$ & $\mathrm{M}$ & 2S180-1 (adg PI 545930; Cochabamba, Bolivia) $\times 10 \mathrm{H} 17$ & + & + & + & + & + & + \\
\hline $12 \mathrm{H} 208-3 \mathrm{~S}$ & W & 2S188-2 (adg PI 546025; Cochabamba, Bolivia) $\times 10 \mathrm{H} 17$ & + & - & + & + & - & + \\
\hline $14 \mathrm{H} 116-1$ & $\mathrm{P}$ & 10H17 × 12H7-2 (adg PI 161683; Tlaxcala, Mexico) & + & + & - & - & - & + \\
\hline $14 \mathrm{H} 116-2$ & $\mathrm{P}$ & 10H17 × 12H7-2 (adg PI 161683; Tlaxcala, Mexico) & - & - & - & - & - & - \\
\hline $14 \mathrm{H} 117-2$ & $\mathrm{P}$ & $10 \mathrm{H} 17 \times 12 \mathrm{H} 12-1$ (adg PI 195162; Huanuco, Peru) & + & + & + & + & + & - \\
\hline 14H117-3S & $\mathrm{P}$ & $10 \mathrm{H} 17 \times 12 \mathrm{H} 12-1$ (adg PI 195162; Huanuco, Peru) & + & - & + & + & + & - \\
\hline $14 \mathrm{H} 119-2$ & $\mathrm{P}$ & $10 \mathrm{H} 17 \times 12 \mathrm{H} 16-1 \mathrm{~S}$ (adg PI 214424; Huanuco, Peru) & + & + & - & - & - & + \\
\hline $14 \mathrm{H} 123-2$ & $\mathrm{P}$ & $10 \mathrm{H} 17 \times 12 \mathrm{H} 34-1$ (adg PI 232840; Cuzco, Peru) & + & + & + & + & + & + \\
\hline $14 \mathrm{H} 126-1 \mathrm{H}$ & $\mathrm{P}$ & $10 \mathrm{H} 17 \times 12 \mathrm{H} 51-1$ (adg PI 243415; Cauca, Colombia) & - & + & - & - & - & - \\
\hline $14 \mathrm{H} 132-1$ & $\mathrm{P}$ & $10 \mathrm{H} 17 \times 12 \mathrm{H} 57-1$ (adg PI 243436; Nortre de Santander, Colombia) & + & + & - & - & - & + \\
\hline $14 \mathrm{H} 137-2$ & $\mathrm{P}$ & $10 \mathrm{H} 17 \times 12 \mathrm{H} 70-1$ (adg PI 246516; Puno, Peru) & + & + & - & - & - & + \\
\hline $14 \mathrm{H} 138-2$ & $\mathrm{P}$ & $10 \mathrm{H} 17 \times 12 \mathrm{H} 70-2($ adg PI 246516; Puno, Peru) & + & + & - & - & - & - \\
\hline 14H141-1S & $\mathrm{P}$ & $10 \mathrm{H} 17 \times 12 \mathrm{H} 82-2$ (adg PI 258879; Oruro, Bolivia) & - & + & + & + & + & - \\
\hline $14 \mathrm{H} 143-2$ & $\mathrm{P}$ & $10 \mathrm{H} 17 \times 12 \mathrm{H} 88-2$ (adg PI 258936; La Paz, Bolivia) & + & + & - & - & - & - \\
\hline 14H161-3S & $\mathrm{P}$ & $10 \mathrm{H} 17 \times 12 \mathrm{H} 138-2$ (adg PI 473249; Jujuy, Argentina) & - & + & - & - & - & - \\
\hline $14 \mathrm{H} 163-1$ & A & 12H6-2 (adg PI 161350; Puebla, Mexico) × 10H17 & + & + & + & + & + & + \\
\hline 14H164-3S & A & 12H7-1 (adg PI 161683; Tlaxcala, Mexico) × 10H17 & + & - & + & + & + & + \\
\hline $14 \mathrm{H} 178-2$ & A & 12H67-1 (adg PI 246499; Ancash, Peru) × 10H17 & + & + & + & + & + & + \\
\hline $14 \mathrm{H} 190-1$ & M & 12H105-2 (adg PI 281031; Oruro, Bolivia) × 10H17 & + & + & + & + & + & - \\
\hline 14H191-3S & A & 12H109-2 (adg PI 281060; Huanuco, Peru) × 10H17 & + & - & - & - & - & - \\
\hline
\end{tabular}


Table 1 (continued)

\begin{tabular}{|c|c|c|c|c|c|c|c|c|}
\hline Clone & Cytoplasm & Pedigree (female $\times$ male); accession and origin of the Andigena parent & $H 1$ & $R 1$ & $R x 1$ & $1 \mathrm{Rx}^{1)}$ & $5 \mathrm{R} \times 1^{1)}$ & $R y_{c h}$ \\
\hline 14H191-4H & A & 12H109-2 (adg PI 281060; Huanuco, Peru) × 10H17 & + & + & + & + & + & + \\
\hline $15 \mathrm{H} 66-2$ & $\mathrm{P}$ & 10H17× 14H52-1 (adg PI 473270; Salta, Argentina) & - & - & + & + & + & - \\
\hline $15 \mathrm{H} 66-4 \mathrm{H}$ & $\mathrm{P}$ & 10H17 × 14H52-1 (adg PI 473270; Salta, Argentina) & + & + & + & + & + & - \\
\hline $15 \mathrm{H} 67-1$ & $\mathrm{P}$ & 10H17× 14H53-1 (adg PI 473275; Jujuy, Argentina) & - & + & + & + & + & + \\
\hline $15 \mathrm{H} 67-2$ & $\mathrm{P}$ & $10 \mathrm{H} 17 \times 14 \mathrm{H} 53-1$ (adg PI 473275; Jujuy, Argentina) & + & + & + & + & + & - \\
\hline $15 \mathrm{H} 69-1 \mathrm{~S}$ & $\mathrm{P}$ & 10H17 × 14H54-2 (adg PI 473276; Jujuy, Argentina) & - & + & + & + & + & - \\
\hline $15 \mathrm{H} 71-1 \mathrm{~S}$ & $\mathrm{P}$ & 10H17 × 14H68-1 (adg PI 473293; Jujuy, Argentina) & + & + & + & + & + & - \\
\hline $15 \mathrm{H} 71-2 \mathrm{H}$ & $\mathrm{P}$ & 10H17 × 14H68-1 (adg PI 473293; Jujuy, Argentina) & + & + & + & + & + & + \\
\hline $15 \mathrm{H} 72-3 \mathrm{H}$ & $\mathrm{P}$ & 10H17 × 14H73-1S (adg PI 473299; Salta, Argentina) & + & + & - & - & - & + \\
\hline $16 \mathrm{H} 51-1$ & $\mathrm{P}$ & $10 \mathrm{H} 17 \times 14 \mathrm{H} 42-1$ (adg PI 473255; Jujuy, Argentina) & + & - & + & + & - & - \\
\hline $16 \mathrm{H} 51-2$ & $\mathrm{P}$ & $10 \mathrm{H} 17 \times 14 \mathrm{H} 42-1$ (adg PI 473255; Jujuy, Argentina) & + & + & + & + & - & + \\
\hline $16 \mathrm{H} 53-1$ & $\mathrm{P}$ & $10 \mathrm{H} 17 \times 14 \mathrm{H} 48-1$ (adg PI 473265; Jujuy, Argentina) & + & + & + & + & + & + \\
\hline $16 \mathrm{H} 53-2$ & $\mathrm{P}$ & $10 \mathrm{H} 17 \times 14 \mathrm{H} 48-1$ (adg PI 473265; Jujuy, Argentina) & + & + & + & + & - & + \\
\hline $16 \mathrm{H} 54-1$ & $\mathrm{P}$ & $10 \mathrm{H} 17 \times 14 \mathrm{H} 48-2$ (adg PI 473265; Jujuy, Argentina) & + & + & + & + & - & + \\
\hline $16 \mathrm{H} 55-2$ & $\mathrm{P}$ & $10 \mathrm{H} 17 \times 14 \mathrm{H} 50-1$ (adg PI 473268; Salta, Argentina) & + & + & + & + & - & - \\
\hline $16 \mathrm{H} 56-2$ & $\mathrm{P}$ & $10 \mathrm{H} 17 \times 14 \mathrm{H} 52-2$ (adg PI 473270; Salta, Argentina) & + & + & + & + & + & + \\
\hline $16 \mathrm{H} 57-2 \mathrm{~S}$ & $\mathrm{P}$ & 10H17 × 14H52-3S (adg PI 473270; Salta, Argentina) & + & - & + & + & - & + \\
\hline $16 \mathrm{H} 60-1 \mathrm{H}$ & $\mathrm{P}$ & 10H17× 14H61-1 (adg PI 473285; Salta, Argentina) & - & + & + & + & + & - \\
\hline $16 \mathrm{H} 60-2 \mathrm{~S}$ & $\mathrm{P}$ & 10H17 × 14H61-1 (adg PI 473285; Salta, Argentina) & + & + & + & + & + & + \\
\hline $16 \mathrm{H} 61-1 \mathrm{H}$ & $\mathrm{P}$ & $10 \mathrm{H} 17 \times 14 \mathrm{H} 61-3 \mathrm{~S}$ (adg PI 473285; Salta, Argentina) & - & + & - & - & - & - \\
\hline $16 \mathrm{H} 62-1 \mathrm{H}$ & $\mathrm{P}$ & 10H17 × 14H64-1S (adg PI 473288; Salta, Argentina) & + & + & - & - & - & - \\
\hline $16 \mathrm{H} 64-1$ & $\mathrm{P}$ & 10H17× 14H69-1 (adg PI 473294; Jujuy, Argentina) & + & + & + & + & + & + \\
\hline $16 \mathrm{H} 64-2$ & $\mathrm{P}$ & 10H17× 14H69-1 (adg PI 473294; Jujuy, Argentina) & - & - & - & - & - & - \\
\hline $16 \mathrm{H} 74-1 \mathrm{~S}$ & $\mathrm{P}$ & 10H17× 14H76-2 (adg PI 473302; Salta, Argentina) & + & + & - & - & - & - \\
\hline $16 \mathrm{H} 77-1$ & $\mathrm{P}$ & 10H17 × 14H79-1H (adg PI 473391; Cochabamba, Bolivia) & + & + & + & + & + & - \\
\hline $16 \mathrm{H} 77-4 \mathrm{H}$ & $\mathrm{P}$ & 10H17 × 14H79-1H (adg PI 473391; Cochabamba, Bolivia) & + & + & + & + & - & - \\
\hline $16 \mathrm{H} 79-2 \mathrm{~S}$ & $\mathrm{P}$ & $10 \mathrm{H} 17 \times 14 \mathrm{H} 81-4 \mathrm{H}$ (adg PI 473508; Chuquisaca, Bolivia) & + & + & + & + & + & + \\
\hline $16 \mathrm{H} 79-3 \mathrm{H}$ & $\mathrm{P}$ & $10 \mathrm{H} 17 \times 14 \mathrm{H} 81-4 \mathrm{H}$ (adg PI 473508; Chuquisaca, Bolivia) & + & + & + & + & - & - \\
\hline $16 \mathrm{H} 81-1$ & $\mathrm{P}$ & 10H17 × 14H84-3S (adg PI 498291; Chuquisaca, Bolivia) & + & + & + & + & + & + \\
\hline $16 \mathrm{H} 83-2$ & $\mathrm{P}$ & 10H17 × 14H87-1 (adg PI 498309; Potosi, Bolivia) & + & - & + & + & + & - \\
\hline $16 \mathrm{H} 85-1$ & $\mathrm{P}$ & 10H17 × 14H101-3H (adg PI 546025; Cochabamba, Bolivia) & + & - & + & + & + & + \\
\hline $16 \mathrm{H} 85-2$ & $\mathrm{P}$ & 10H17 × 14H101-3H (adg PI 546025; Cochabamba, Bolivia) & + & + & + & + & - & - \\
\hline $16 \mathrm{H} 85-3 \mathrm{~S}$ & $\mathrm{P}$ & 10H17 × 14H101-3H (adg PI 546025; Cochabamba, Bolivia) & + & + & + & + & + & - \\
\hline $16 \mathrm{H} 85-4 \mathrm{H}$ & $\mathrm{P}$ & 10H17 × 14H101-3H (adg PI 546025; Cochabamba, Bolivia) & + & + & + & + & + & - \\
\hline $16 \mathrm{H} 86-1$ & $\mathrm{P}$ & $10 \mathrm{H} 17 \times 14 \mathrm{H} 102-1$ (adg PI 546026; Chuquisaca, Bolivia) & + & + & - & - & - & - \\
\hline $16 \mathrm{H} 90-1$ & $\mathrm{~W}$ & 14H61-1 (adg PI 473285; Salta, Argentina) × 10H17 & + & + & + & + & + & + \\
\hline $16 \mathrm{H} 91-3 \mathrm{~S}$ & A & 14H69-1 (adg PI 473294; Jujuy, Argentina) × 10H17 & - & + & + & + & + & - \\
\hline $16 \mathrm{H} 92-1$ & A & 14H74-1 (adg PI 473300; Salta, Argentina) × 10H17 & + & + & + & + & + & - \\
\hline $16 \mathrm{H} 93-2$ & A & 14H84-2 (adg PI 498291; Chuquisaca, Bolivia) × 10H17 & + & + & + & + & + & - \\
\hline $16 \mathrm{H} 100-1$ & $\mathrm{~T}$ & 4H117-2 [4H108-3 (tar PI 473245) × 2S70 (adg PI 258917)] $\times 10 \mathrm{H} 17$ & + & + & - & - & - & + \\
\hline $16 \mathrm{H} 102-1$ & $\mathrm{~T}$ & 4H122-1 [4H109-3 (tar PI 473244) × 2S156 (adg PI 473294)] × 10H17 & - & + & + & + & + & + \\
\hline $16 \mathrm{H} 102-2$ & $\mathrm{~T}$ & 4H122-1 [4H109-3 (tar PI 473244) × 2S156 (adg PI 473294)] × 10H17 & + & + & + & + & + & + \\
\hline $16 \mathrm{H} 107-1$ & $\mathrm{~T}$ & 4H125-4 [4H109-7 (tar PI 473244) × 2S156 (adg PI 473294)] × 10H17 & - & + & + & + & + & + \\
\hline $16 \mathrm{H} 107-3$ & $\mathrm{~T}$ & 4H125-4 [4H109-7 (tar PI 473244) × 2S156 (adg PI 473294)] × 10H17 & + & + & + & + & + & - \\
\hline $16 \mathrm{H} 108-2$ & $\mathrm{~T}$ & 4H125-5 [4H109-7 (tar PI 473244) × 2S156 (adg PI 473294)] × 10H17 & + & + & + & + & - & + \\
\hline $16 \mathrm{H} 115-1$ & $\mathrm{P}$ & $10 \mathrm{H} 17 \times 4 \mathrm{H} 125-5$ [4H109-7 (tar PI 473244)× 2S156 (adg PI 473294)] & + & + & + & + & - & - \\
\hline $16 \mathrm{H} 115-2$ & $\mathrm{P}$ & $10 \mathrm{H} 17 \times 4 \mathrm{H} 125-5$ [4H109-7 (tar PI 473244) × 2S156 (adg PI 473294)] & + & + & + & + & + & - \\
\hline
\end{tabular}

1) PCR markers $1 \mathrm{Rx} 1$ and 5Rx1 identify Exon 1 and the promotor region of $R x 1$ gene, respectively (Ahmadvand et al. 2013) 


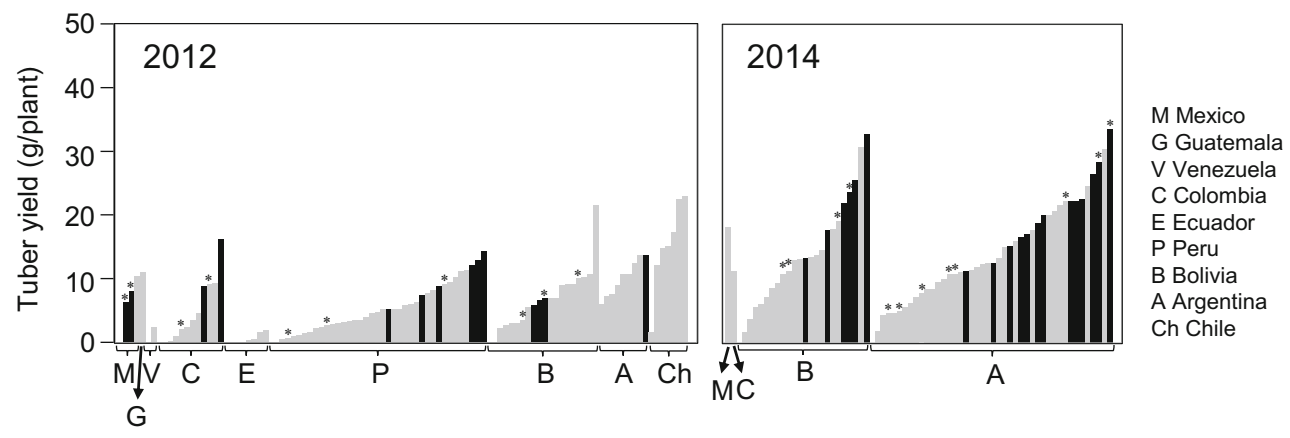

Fig. 2 Tuber yields (g/plant) in Andigena accessions for the second selection cycle in 2012 and the third selection cycle in 2014. The Andigena accessions deriving PGEL clones are shown by black bars.

was derived from $S$. tarijense. All these were male fertile as these were used as pollen parents to cross with Atlantic.

PGEL clones tend to be late-maturing due to the effect of Andigena and have relatively short tuber dormancy because the maternal progenitor of $10 \mathrm{H} 17$ was $S$. phureja (Mori et al. 2012), a diploid cultivated species with short or no tuber dormancy (Hawkes 1990), which is dominant over long dormancy (Freyre et al. 1994).

\section{Informative SNPs}

The 22 K SNP array genotyped 21,027 SNP loci for 95 PGEL clones, 24 varieties, 10H17, and DM. From this dataset, heterozygous SNPs in the DM clone were first discarded (451 SNPs). Then, ambiguous SNP loci were discarded (228 SNPs) based on "Bad and questionable SNPs $(n=637)$ ", "MSUPBG remove (n=399)", "more or less than 1 hit $(n=22)$ ", and "CNV filter $(n=185)$ " files provided in Peterson et al. (2016). Then, SNPs with $>10 \%$ missing values (372 SNPs) and monomorphic SNPs (2029 SNPs) were discarded. Finally, 17,947 informative SNPs were selected. Using the $8 \mathrm{~K}$ SNP array, Hirsch et al. (2013) selected 6373 SNPs for analyzing the diversity panel of North American varieties. A total of 462 genotypes, including Hirsch et al. (2013)'s 247 genotypes, almost all Japanese varieties (Igarashi et al. 2019), and
The PGEL clones obtained from the first selection cycle were derived from the Andigena accessions shown with asterisks

PGEL clones, were used to select informative SNPs from the 6373 SNPs according to the same procedure described above, which resulted in 5707 informative SNPs.

\section{Relationship of PGEL Clones with the Other Varieties}

Using 5707 SNPs for 462 genotypes or 17,947 SNPs for 120 genotypes, UPGMA dendrograms were constructed (Fig. 4) and the mean Euclidean distances within and between populations were calculated (Table 3). In both dendrograms, 95 PGEL clones and their common parent 10H17 formed a distinct cluster, while $4 x$ varieties and breeding clones formed several other clusters. These clusters were first united together and then with more exotic germplasm such as $S$. phurejabased diploid varieties, genetic stocks, and wild species in the 5707 SNP dendrogram or with DM in the 17,947 SNP dendrogram (Fig. 4). Using 5707 SNPs, the mean Euclidean distance among 95 PGEL clones was 41.6 (Table 3), which was significantly lower than those within Japanese or foreign $4 x$ varieties and breeding clones ( 44.4 or 48.4 , respectively) because PGEL clones were descended from the same parent 10H17. However, interpopulation distances of PGEL clones were larger with Japanese, foreign, or all $4 x$ genotypes (48.0, 49.9 or 49.4 , respectively) than the distance between Japanese and foreign genotypes (47.2). Wild and genetic stocks were

Table 2 Differences in tuber yield between populations grown in the same year

\begin{tabular}{|c|c|c|c|c|c|c|c|c|c|c|c|c|}
\hline \multirow[t]{2}{*}{ Population } & \multicolumn{3}{|c|}{2012} & \multicolumn{3}{|c|}{2014} & \multicolumn{3}{|c|}{2015} & \multicolumn{3}{|c|}{2016} \\
\hline & $\mathrm{n}$ & Mean (SD) & Range & $\mathrm{n}$ & Mean (SD) & Range & $\mathrm{n}$ & Mean (SD) & Range & $\mathrm{n}$ & Mean (SD) & Range \\
\hline Andigena & 102 & $6.2(5.28)$ & $0.0-22.8$ & 70 & $14.2(7.99)$ & $0.0-33.5$ & & & & & & \\
\hline $\mathrm{F}_{1}($ Andigena $\times 10 \mathrm{H} 17)$ & 69 & $28.4(6.00)$ & $11.9-52.8$ & 83 & $28.2(6.89)$ & $15.7-46.7$ & 16 & $19.3(4.07)$ & $12.6-27.1$ & 50 & $26.0(5.42)$ & $14.5-38.4$ \\
\hline Atlantic $\times \mathrm{F}_{1}$ hybrid & & & & & & & 48 & $29.9(6.40)$ & $16.9-41.7$ & 36 & $31.8(5.16)$ & $21.9-44.6$ \\
\hline Control $^{1)}$ & & & & & 35.1 & & & 20.4 & & & 31.7 & \\
\hline$t$ test & & $P<0.0001$ & & & $P<0.0001$ & & & $P<0.0001$ & & & $P<0.0001$ & \\
\hline
\end{tabular}

The number of families, the populational mean tuber yield $(\mathrm{g} / \mathrm{plant})$, and the range of familial means for each population are represented

1) A seedling population of $\mathrm{F}_{1}$ (Atlantic $\left.\times 10 \mathrm{H} 17\right)$ 


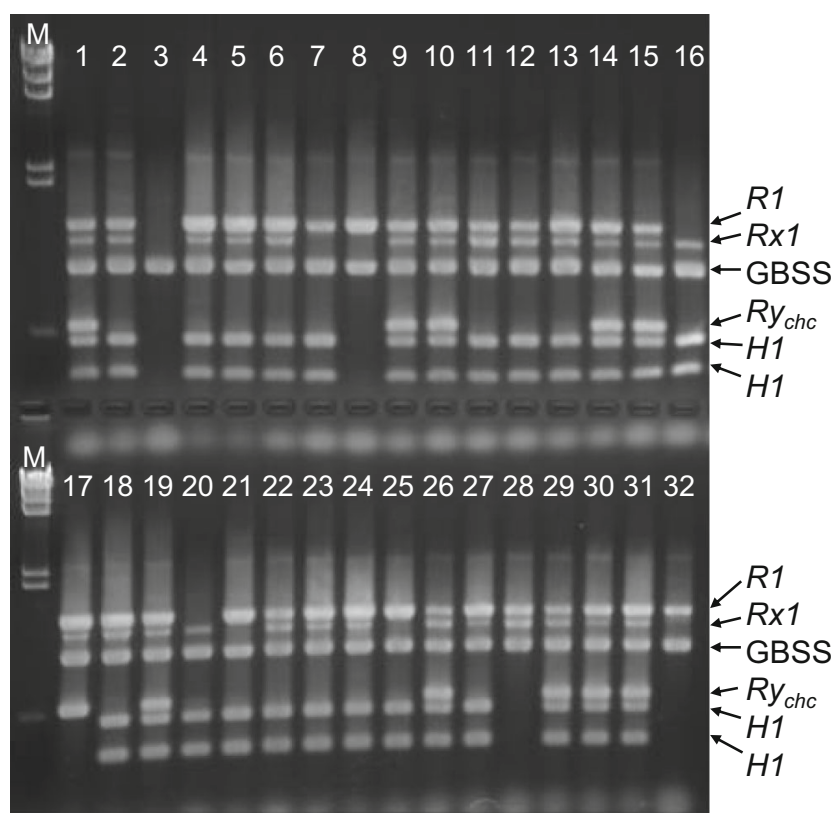

Fig. 3 Characterization of PGEL clones using the multiplex PCR method (Mori et al. 2011). M, lambda DNA HindIII digest; 1, 16H54-1; 2, 16H79-3H; 3, 16H64-2; 4, 16H77-4H; 5, 16H92-1; 6, 16H77-1; 7, 16H62-1H; 8, 16H61-1H; 9, 16H90-1; 10, 16H64-1; 11, 16H107-3; $12,16 \mathrm{H} 115-1 ; 13,16 \mathrm{H} 93-2 ; 14,16 \mathrm{H} 102-2 ; 15,16 \mathrm{H} 79-2 \mathrm{~S} ; 16$, 16H83-2; 17, 16H107-1; 18, 16H115-2; 19, 16H56-2; 20, 16H51-1; $21,16 \mathrm{H} 86-1 ; 22,16 \mathrm{H} 85-3 \mathrm{~S} ; 23,16 \mathrm{H} 85-2 ; 24,16 \mathrm{H} 85-4 \mathrm{H} ; 25,16 \mathrm{H} 74$ 1S; 26, 16H60-2S; 27, 16H55-2; 28, 16H60-1H; 29, 16H53-1; 30, 16H53-2; 31, 16H81-1; 32, cv. Toyoshiro

closer to PGEL clones (54.6) than to $4 x$ varieties and breeding clones (56.4). A similar trend was found using 17,947 SNPs (Table 3): interpopulation distances between PGEL clones and Japanese or foreign varieties were larger than that between Japanese and foreign varieties, and DM was closer to PGEL clones than to $4 x$ varieties.

\section{New Alleles from PGEL Clones}

Using the $8 \mathrm{~K}$ SNP array, 409 SNPs $(7.4 \%$ of informative SNPs) were polymorphic among 25 varieties, including 10H17, but monomorphic among 95 PGEL clones, while 40 SNPs $(0.7 \%$ of informative SNPs) were polymorphic among PGEL clones but monomorphic among 24 varieties (Supplementary Table 3). A very low incidence of PGEL clone-specific SNPs was expected because the $8 \mathrm{~K}$ SNPs were originally developed as a collection of polymorphic nucleotides among only six varieties (Hamilton et al. 2011). The $22 \mathrm{~K} \mathrm{SNP}$ array is composed of informative SNPs among the six varieties and those of 83 diverse collections detected by Uitdewilligen et al. (2013). Using the $22 \mathrm{~K} \mathrm{SNP}$ array, $7.8 \%$ and $8.4 \%$ of informative SNPs were polymorphic only among PGEL clones and among the 25 varieties, respectively.

\section{Discussion}

The breeding procedures applied in this study were based on the following assumptions: a high-yielding parent can generate high-yielding progeny, and the tuber-yielding ability of a genotype can be evaluated by the mean tuber yield of the derived seedling population. There are many controversies in these assumptions, such as that the heterotic effect may overcome an additive effect in yield and that tuber yield in seedlings may not be correlated with field performance (Bradshaw and Mackay 1994). However, in this study, superior genotypes were selected not as commercial varieties but as parental lines, which require good general combining ability (Bradshaw and Mackay 1994). Furthermore, tuber yields in seedling populations of Atlantic $\times \mathrm{F}_{1}$ hybrid in the first selection cycle were weakly, but positively correlated with those of their field-grown clonal populations $(r=0.388$, unpublished data). Therefore, considering a short period of time to incorporate a wider range of genetic variation, these assumptions had to be accepted.

\section{Long-Day-Adapted Clones Were Created from Andigena}

Neo-Tuberosum was developed from Andigena by four to six cycles of recurrent selection primarily for long-day-length adaptation as expressed by tuber production (Glendinning 1975b; Rasco et al. 1980). We developed 95 long-dayadapted tetraploid clones (PGEL clones) derived from 49 Andigena accessions and four $4 x$ tar-adg hybrids. These are all $\mathrm{F}_{1}$ hybrids of Andigena or $4 x$ tar-adg hybrids with $10 \mathrm{H} 17$.

Andigena is geographically differentiated (Salaman 1946; Glendinning 1968; Sukhotu et al. 2005), and a preliminary report indicated that the tuber-yielding ability of Andigena under northern temperate regions was also geographically differentiated (Hosaka and Hanneman1993). The same tendency was observed in the selection experiment of Andigena performed in 2012 (Fig. 2). This was a reason why we first selected long-day-adapted Andigena genotypes (step 1) and then crossed them with $10 \mathrm{H} 17$ (step 2). However, PGEL clones were not always derived from high-yielding Andigena accessions under long days. This suggests that adaptability to day-length in Andigena is geographically differentiated, but tuber-yielding ability under long days might be acquired to some extent by a heterotic effect that occurs by hybridization between Andigena and common potatoes. Heterosis in tuber yield, as discussed below, has been described in the $F_{1}$ hybrids between long-day-adapted Andigena and common potato varieties (Glendinning 1969; Cubillos and Plaisted 1976; Tarn and Tai 1977; Maris 1989). Therefore, both strategies in which Andigena is first hybridized with common 
potatoes and then selected for long-day adaptability and in which it is first selected for long-day adaptability and then hybridized with common potatoes can successfully obtain long-day-adapted tetraploid clones.

\section{Broadening Genetic Diversity Using PGEL Clones}

By cluster analysis, PGEL clones formed a unique cluster distinct from a worldwide collection of commercial varieties and from primitive Andean cultivated potatoes and wild species (Fig. 4). Interpopulation distance between PGEL clones and all $4 x$ varieties was larger (49.4) than the distance among all $4 x$ varieties (47.6), and the distance of wild and genetic stocks was closer to PGEL clones than to $4 x$ varieties (54.6 and 56.4, respectively) (Table 3). In addition, an almost equal number of new SNPs was detected among PGEL clones compared with SNPs specifically found among 25 diverse varieties. PGEL clones are all male fertile, which enables their use as pollen parents in breeding programs. Female fertility
Fig. 4 UPGMA dendrograms using 1) 5707 SNPs for 462 genotypes or 2) 17,947 SNPs for 120 genotypes
1) $5707 \mathrm{SNPs}$

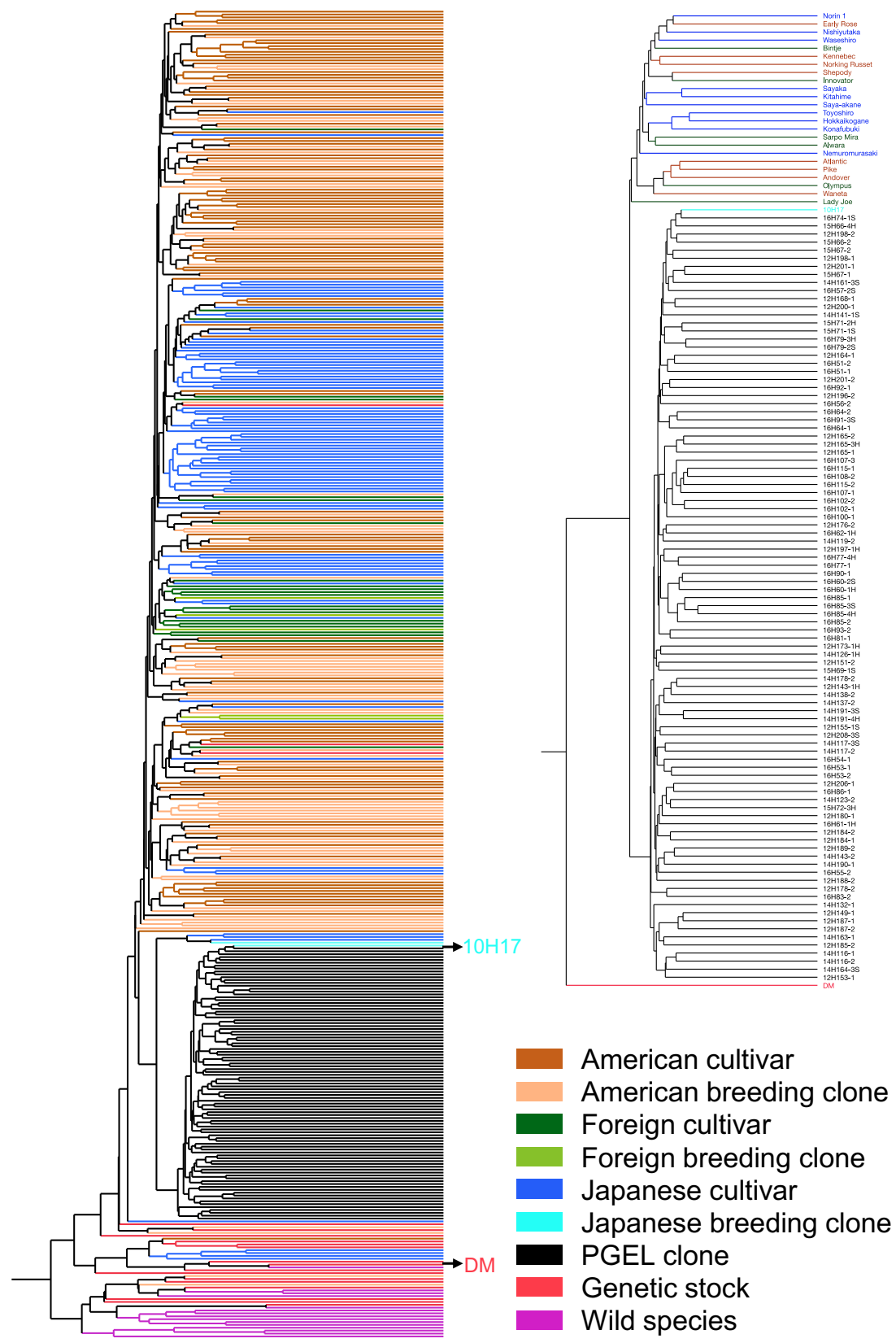


Table 3 Mean Euclidean distances within and between populations (standard deviation in parentheses)

\begin{tabular}{|c|c|c|c|c|c|c|}
\hline Population & $\mathrm{n}$ & PGEL clones & $4 x$ varieties & Foreign $4 x$ varieties & Japanese $4 x$ varieties & $\begin{array}{l}\text { Wild and genetic stocks } \\
\text { DM }\end{array}$ \\
\hline \multirow[t]{2}{*}{ PGEL clones } & 95 & $41.6(1.70)$ & & & & \\
\hline & 95 & $71.2(2.73)$ & & & & \\
\hline \multirow[t]{2}{*}{$4 x$ varieties } & 326 & $49.4(1.63)$ & $47.6(2.44)$ & & & \\
\hline & 24 & $81.5(2.12)$ & $76.9(4.52)$ & & & \\
\hline \multirow[t]{2}{*}{ Foreign $4 x$ varieties } & 240 & $49.9(1.46)$ & & $48.4(2.33)$ & & \\
\hline & 14 & $82.1(2.17)$ & & $78.0(5.19)$ & & \\
\hline \multirow[t]{2}{*}{ Japanese $4 x$ varieties } & 86 & $48.0(1.27)$ & & $47.2(2.00)$ & $44.4(2.29)$ & \multirow{4}{*}{$49.5(7.42)$} \\
\hline & 10 & $80.8(1.81)$ & & $77.2(3.25)$ & $73.8(5.14)$ & \\
\hline Wild and genetic stocks & 34 & $54.6(3.41)$ & $56.4(4.11)$ & $56.6(4.08)$ & $55.9(4.15)$ & \\
\hline $\mathrm{DM}$ & 1 & $95.7(1.75)$ & $100.0(2.35)$ & $100.4(2.98)$ & $99.5(0.91)$ & \\
\hline
\end{tabular}

Upper column, using 5707 SNPs for 462 genotypes

Lower column, using 17,947 SNPs for 120 genotypes

has also been confirmed for most of the PGEL clones (data not shown), which facilitates the introduction of cytoplasmic genome diversity. Thus, the use of PGEL clones would increase genetic diversity in the breeder's gene pool.

\section{PGEL Clones Are Also Useful for Resistance Breeding}

Of the 95 PGEL clones, 23 had all four resistance genes and were useful for resistance breeding. Extreme resistance to Potato virus $X$ has frequently been noted in Andigena clones, and $R x 1$ was derived from Andigena CPC1673 (Cockerham 1970; Ritter et al. 1991). Therefore, overrepresentation of $R x 1$ among PGEL clones could be explained by $R x l$ derived from $10 \mathrm{H} 17$ and from some Andigena parents. In fact, 12 PGEL clones did not have the $5 \mathrm{Rx} 1$ marker (promotor) but did have the 1Rx1 (Exon 1) and Rx1 markers (Exon 1 and a spacer region, detected by the multiplex PCR method in this study), suggesting that their $R x 1$ gene was not derived from $10 \mathrm{H} 17$ but from Andigena, and it is not known whether it functionally expresses the resistance. The dosages of resistance genes in $10 \mathrm{H} 17$ are estimated to be simplex for $R x 1$ and $R y_{c h c}$ and duplex for $H 1$ and $R 1$. Because both parents, Saikai 35 and Pike, have the $H 1$ gene, its duplex status in $10 \mathrm{H} 17$ is highly probable. However, $R 1$ was derived unilaterally from Pike into $10 \mathrm{H} 17$ (Fujimatsu et al. 2018) and is less likely to be found among Andigena accessions because $R 1$ was originally derived from a Mexican hexaploid wild species $S$. demissum (Ross 1986). The $R 1$ marker band was also significantly overrepresented in the population of Saikai $35 \times$ Pike, to which poor PCR conditions were suggested (Fujimatsu et al. 2018). The most likely explanation would be a genetic linkage between $R 1$ and either $H 1$ or a maturity-related trait. $R 1$ and $H 1$ are localized on different arms of chromosome 5, while both $R 1$ and a major QTL for plant maturity or a tuberizationregulating gene (StCDF1) are localized to the distal end of the short arm of chromosome 5 (Leonards-Schippers et al. 1992;
Pineda et al. 1993; Kloosterman et al. 2013; Massa et al. 2015). Considering the relatively high discordance in the presence/absence of $R 1$ and that of $H 1$ (26 of 95 clones), cosegregation of $R l$ with the maturity trait is more likely to cause overrepresentation of $R l$ because early maturity is a substantial requirement to obtain high tuber yields under long days. Note that our recent analysis using real-time PCR indicated $10 \mathrm{H} 17$ is duplex for $H 1$, but simplex for $R x I$ and $R I$ (data not shown).

\section{Conclusion}

To broaden the genetic diversity, Andigena was utilized through a single event of hybridization, from which longday-adapted $F_{1}$ hybrid clones were selected. After evaluating these $\mathrm{F}_{1}$ hybrids as pollen parents, $95 \mathrm{~F}_{1}$ hybrid clones were selected and named PGEL clones, which were released to breeders. The mean tuber yield among families of Atlantic $\times$ long-day-adapted $F_{1}$ hybrid was significantly higher than that among $\mathrm{F}_{1}$ hybrid families of Andigena $\times 10 \mathrm{H} 17$ (Table 2), and most of the families of Atlantic $\times$ PGEL clones outyielded the control family (Supplementary Fig. 2). Thus, using PGEL clones as parents in commercial breeding, a heterotic effect for tuber yield can be expected. An additional advantage of the PGEL clones is disease resistance, which can be managed by molecular markers. However, since total tuber yield was a primary selection criterion, other agronomic traits, such as tuber appearance, stolon length, and maturity, are still poor. Improving general agronomic traits and stacking multiple resistance genes in multiplex conditions are underway.

Acknowledgements We thank Akito Nashiki for giving us a preliminary result using 1Rx and 5Rx1 markers, and Shizuka Souma and Eri Kakuta for technical assistance. This research was carried out with full financial support from Calbee Inc., Hokkaido Potato Growers Association, Kewpie Corp., KENKO Mayonnaise Co., Ltd., and Japan Snack Cereal Foods Association. 


\section{Appendix (Breeding Process)}

\section{First Selection Cycle}

Step 1: For the first selection cycle, the Andigena genotypes used were the ones maintained as tubers in our laboratory. Each genotype was derived from a different Plant Introduction (PI)-numbered Andigena accession and was not selected for any traits before.

Step 2: One tuber each of 127 PI-numbered Andigena accessions was planted on September 4, 2011. From November 2011 to February 2012, 88 Andigena genotypes flowered and were pollinated reciprocally with $10 \mathrm{H} 17$. From a total of 1400 pollinations, seeds were obtained from 33 Andigena genotypes when used as female parents and 36 Andigena genotypes when used as male parents. Fifteen Andigena genotypes were successful both as female and male parents.

Step 3: The seeds of these 69 hybrid families were sown on April 15-16, 2012. A total of 2005 seedlings with a maximum of 30 seedlings per accession were transplanted on May 5-6, and the tubers were harvested on August 14-21. From the top 40 families in tuber yields, the best-looking two genotypes in each family were selected. In addition, 10 genotypes each were selected by our preferences (genotypes with the identity suffixed with $\mathrm{H}$ or $\mathrm{S}$ were preferred by $\mathrm{K}$. Hosaka or R. Sanetomo, respectively). As a result, 100 genotypes of 49 families derived from 38 Andigena accessions were selected as long-day-adapted $F_{1}$ hybrids (Supplementary Fig. 1).

Step 4: The tubers of the 100 genotypes were planted on November 13, 2012. During January and February 2013, 88 genotypes flowered and produced pollen, which were crossed as pollen parents with Atlantic. By pollinating a total of 1018 flowers, 627 berries were obtained from 79 pollen parents.

Step 5: Of the 79 cross combinations (=families), two families were accidently contaminated; thus, the seeds of 77 families were sown on May 1, 2013. A total of 2310 seedling plants with a maximum of 30 seedlings per accession were transplanted on May 24-25, and the tubers were harvested on August 27-30. Based on tuber yields, the highest 30 families were identified and their parental $F_{1}$ hybrids were regarded as superior long-dayadapted $F_{1}$ hybrids. Note that one of the highyielding parents was lost during storage, so the 31 st genotype was selected (Supplementary Fig. 2).

\section{Second Selection Cycle}

Step 1: The seeds of 135 PI-numbered Andigena accessions were sown on April 11-12, 2012. A maximum of 15 plants per accession were transplanted on May 2-3 and May 15, resulting in a total of 1242 seedlings of the 105 accessions. These were grown outside without any physical protection. Tubers were harvested on August 14-21. From 50 high-yielding accessions, the best-looking two genotypes from each accession were selected. In addition, 20 genotypes were selected based on our preferences. As a result, 120 genotypes of 59 Andigena accessions were selected as long-day-adapted Andigena genotypes (Fig. 1).

Step 2: The tubers of 119 Andigena genotypes (one dried up during cold storage) were planted on October 16, 2013. By early February 2014, 79 genotypes flowered and crossed reciprocally with $10 \mathrm{H} 17$. Pollinations on 1173 flowers of 150 cross combinations produced 429 berries from 97 cross combinations. Seventeen, 30, and 25 Andigena genotypes were successfully crossed only as female parents, only as male parents, and as both female and male parents, respectively. However, an accidental shutdown of the heating system on February 4, 2014, killed almost all plants in the greenhouse and stopped maturation of the berries setting on the plants.

Step 3: Because of the freezing accident, some berries were prematured and contained only too small seeds. Thus, a sufficient number of seeds was obtained from 83 out of the 97 successful cross combinations. These were sown on April 28, 2014. On May 16-27, 2449 seedlings of the 83 families were transplanted, and the tubers were harvested on August 21-27. From this season, a control family ( $\mathrm{F}_{1}$ hybrid seedlings of Atlantic $\times 10 \mathrm{H} 17$ ) was included in the experiment. Fifteen families outyielded the control family ( $35.1 \mathrm{~g} /$ plant) (Supplementary Fig. 1). From these 15 and the second highest-yielding 15 families, the best-looking two genotypes per family were selected. In addition, 20 genotypes were selected based on our preferences. Consequently, 80 genotypes of 39 families derived from 26 PI-numbered Andigena accessions were selected as long-day-adapted $F_{1}$ hybrids.

Step 4: The tubers of the 80 genotypes were planted on October 5, 2014. From mid-November 2014 to early February 2015, 71 genotypes flowered and produced pollen, which were used as pollen parents to cross with Atlantic. By pollinating 700 flowers, 401 berries were obtained from 60 pollen parents. Nineteen unsuccessful genotypes were grown again 
in the fall of 2015. The tubers were planted on October 11, 2015. From 228 pollinations of 18 genotypes with Atlantic, 140 berries were obtained from 14 genotypes.

Step 5: Of the first set of 60 successful cross combinations (=families), 48 families produced matured seeds by the spring season, which were sown on May 6, 2015. The remaining 12 families were not evaluated because the parental long-day-adapted $\mathrm{F}_{1}$ hybrids were erroneously thrown away before their progeny evaluation. A total of 1431 seedling plants were transplanted on May 25-28, and the tubers were harvested on August 27-28. Most of the families outyielded the control family grown this year (20.4 g/plant) (Supplementary Fig. 2). The highest 15 families were identified, and their parents were regarded as superior long-day-adapted $\mathrm{F}_{1}$ hybrids and distributed to breeders. The second set of 14 hybrid families were evaluated in 2016. The seeds were sown on May 5, and 420 seedlings were transplanted on May 25. Tubers were harvested on August 29-31. Eight of 14 families outyielded the control family ( $31.7 \mathrm{~g} / \mathrm{plant})$. In addition to tuber yield, specific gravity was also considered in this season. Based on the higher tuber yield and specific gravity, the highest four families were identified, and their parents were distributed to breeders as superior long-day-adapted $\mathrm{F}_{1}$ hybrids.

\section{Third Selection Cycle}

Step 1: The seeds of 74 PI-numbered Andigena accessions were sown on April 25, 2014. A maximum of 15 plants per accession were transplanted on May 1427 , resulting in a total of 821 seedlings of 70 Andigena accessions. Tubers were harvested on August 21-27. From the 30 highest-yielding accessions, the best-looking two genotypes from each accession were selected (in two accessions, only one genotype each was grown and selected). In addition, 20 genotypes were selected based on our preferences. As a result, 78 genotypes of 37 PInumbered Andigena accessions were selected as long-day-adapted Andigena genotypes (Fig. 1).

Step 2: The tubers of the 78 Andigena genotypes were planted on October 5, 2014. Since the emergence varied by genotype, reciprocal crosses with $10 \mathrm{H} 17$ continued until early May 2015. Sixty-one genotypes flowered and crossed with $10 \mathrm{H} 17$. Pollinations on 1094 flowers of 109 cross combinations produced 219 berries from 55 cross combinations. Four Andigena genotypes were successfully crossed only as female parents, while 29 Andigena genotypes were successfully crossed only as male parents with $10 \mathrm{H} 17$. Eleven Andigena genotypes were successfully crossed reciprocally with $10 \mathrm{H} 17$. The tubers of 19 unsuccessful Andigena genotypes were planted again on October 15 in 2015. One did not emerge, while four genotypes did not flower by mid-March 2016. The remaining 14 genotypes were crossed with 10H17. Pollinations on 473 flowers of 26 cross combinations produced 67 berries from 13 cross combinations. Nine Andigena genotypes were successful only as male parents. Two Andigena genotypes were successfully crossed reciprocally with $10 \mathrm{H} 17$.

Step 3: Of the 55 successful crosses made in 2015,16 produced matured seeds by the spring of 2015. These seeds were sown on May 6, 2015. A total of 477 seedlings of the 16 families were transplanted on May 25-29, and tubers were harvested on August 26. Six families outyielded the control population (20.4 g/plant) (Supplementary Fig. 1). The bestlooking two genotypes in each of six families were selected. In addition, 10 genotypes were selected based on our preferences. Consequently, 22 genotypes of 12 families derived from 11 Andigena accessions were selected as long-day-adapted $F_{1}$ hybrids. The remaining 39 hybrid families and the second set of 13 hybrid families were evaluated in 2016 (Fig. 1). The number of seeds in two families was not sufficient. Thus, the seeds of 50 hybrid families were sown on May 5, and 1499 seedlings were transplanted on May 26. Tubers were harvested on August 29-31. Eight families outyielded the control family (31.7 g/plant) (Supplementary Fig. 1). The best-looking two genotypes in each of 20 highyielding families were selected. In addition, 30 genotypes were selected based on our preferences. As a result, 70 genotypes of 31 families derived from 18 Andigena accessions were selected as long-dayadapted $\mathrm{F}_{1}$ hybrids.

Step 4: After $\mathrm{GA}_{3}$ treatment for breaking tuber dormancy, the tubers of the first set of 22 long-day-adapted $\mathrm{F}_{1}$ hybrids were planted on October 14, 2015. All emerged by early December and crossed as pollen parents with Atlantic. By pollinating 217 flowers, 156 berries were obtained from all 22 genotypes. In 2016, the second set of 70 long-day-adapted $F_{1}$ hybrids was treated with $\mathrm{GA}_{3}$ for breaking tuber dormancy and planted on October 15 . All but one emerged by early January 2017. From 659 pollinations on Atlantic, 368 berries were obtained from 59 male genotypes.

Step 5: The seeds of 22 hybrid families with Atlantic were sown on May 5, 2016 and 660 seedlings were 
transplanted on May 25. Tubers were harvested on August 29-31. Ten of 22 families outyielded the control family (31.7 g/plant) (Supplementary Fig. 2). Based on the higher tuber yield and specific gravity, eight parental $F_{1}$ hybrids were selected and distributed to breeders. The second set of 59 hybrid families with Atlantic was evaluated in 2017. The seeds were sown on May 2, and 1768 seedlings were transplanted on May 24-25. Tubers were harvested on August 28-29. Forty-eight families outyielded the control family (25.5 g/plant). Based on higher tuber yield and specific gravity, 30 families were selected, and their parents were regarded as superior long-day-adapted $\mathrm{F}_{1}$ hybrids and distributed to breeders.

\section{Fourth Selection Cycle}

Ten clones of $4 x$ tar-adg hybrids were used as parental genotypes in this selection cycle. Two PI-numbered S. tarijense and 3 PI-numbered Andigena accessions were involved in these hybrids as female parents and male parents, respectively. Processes of steps 1 and 2 were described elsewhere (Hosaka et al. 2018).

Step 3: Ten families derived from crosses of $4 x$ tar-adg hybrid $\times 10 \mathrm{H} 17$ and seven families from the reciprocal crosses were raised as seedlings in 2016. The seeds were sown on May 5, and 509 seedlings were transplanted on May 26. Tubers were harvested on August 29-31. Only one family outyielded the control family (31.7 g/plant). The best-looking two genotypes in each of seven high-yielding families were selected. In addition, one genotype was selected based on our preferences. A total of 15 genotypes of seven families derived from six clones of $4 x$ tar-adg hybrids were selected as long-day-adapted $\mathrm{F}_{1}$ hybrids.

Step 4: After a $\mathrm{GA}_{3}$ treatment for breaking tuber dormancy, the tubers of the 15 genotypes were planted on October 15, 2016. All emerged by the end of December and were crossed as pollen parents with Atlantic. By pollinating 149 flowers, 73 berries were obtained from 12 male parents.

Step 5: The seeds of the 12 hybrid families with Atlantic were sown on May 2, 2017, and 360 seedlings were transplanted on May 24-25. Tubers were harvested on August 28-29. Eight of the 12 families outyielded the control family (25.5 g/plant) (Supplementary Fig. 2). Thus, the parents of these eight high-yielding families were regarded as superior long-day-adapted $\mathrm{F}_{1}$ hybrids and were distributed to breeders in 2017.
Open Access This article is licensed under a Creative Commons Attribution 4.0 International License, which permits use, sharing, adaptation, distribution and reproduction in any medium or format, as long as you give appropriate credit to the original author(s) and the source, provide a link to the Creative Commons licence, and indicate if changes were made. The images or other third party material in this article are included in the article's Creative Commons licence, unless indicated otherwise in a credit line to the material. If material is not included in the article's Creative Commons licence and your intended use is not permitted by statutory regulation or exceeds the permitted use, you will need to obtain permission directly from the copyright holder. To view a copy of this licence, visit http://creativecommons.org/licenses/by/4.0/.

\section{References}

Ahmadvand, R., I. Wolf, A.M. Gorji, Z. Polgár, and J. Taller. 2013. Development of molecular tools for distinguishing between the highly similar $R x 1$ and $R x 2$ PVX extreme resistance genes in tetraploid potato. Potato Research 56: 277-291.

Akeley, R., W. Mills, C. Cunningham, and J. Watts. 1968. Lenape: A new potato variety high in solids and chipping quality. American Potato Journal 45: 142-145.

Asama, K., H. Ito, N. Murakami, and T. Itoh. 1982. New potato variety "Konafubuki". The Bulletin of Hokkaido Prefectural Agricultural Experiment Station 48: 75-84.

Bendahmane, A., D.C. Baulcombe, and K. Kanyuka. 1999. The Rx gene from potato controls separate virus resistance and cell death responses. Plant Cell 11: 781-791.

Bradshaw, J.E., and G.R. Mackay. 1994. Breeding strategies for clonally propagated potatoes. In Potato genetics, ed. J.E. Bradshaw and G.R. Mackay, 467-497. Wallingford: CAB International.

Cockerham, G. 1970. Genetical studies on resistance to potato viruses X and Y. Heredity 25: 309-348.

Cubillos, A.G., and R.L. Plaisted. 1976. Heterosis for yield in hybrids between $S$. tuberosum ssp. tuberosum and tuberosum ssp. andigena. American Potato Journal 53: 143-150.

Dionne, L.A. 1961. Cytoplasmic sterility in derivatives of Solanum demissum. American Potato Journal 38: 117-120.

Douches, D.S., D. Maas, K. Jastrzebski, and R.W. Chase. 1996. Assessment of potato breeding progress in the USA over the last century. Crop Science 36: 1544-1552.

Ehlenfeldt, M., D. Lopez-Portilla, A. Boe, and R. Johansen. 1990. Reducing sugar accumulation in progeny families of cold chipping potato clones. American Potato Journal 67: 83-91.

Freyre, R., S. Warnke, B. Sosinski, and D.S. Douches. 1994. Quantitative trait locus analysis of tuber dormancy in diploid potato (Solanum spp.). Theoretical and Applied Genetics 89: 474-480.

Fujimatsu, M., H. Hashizume, T. Fudan, Y. Koma, R. Sanetomo, S. Ono, and K. Hosaka. 2018. Harimaru: A new potato variety for a local specialty. Breeding Science 68: 284-288.

Glendinning, D.R. 1968. Regional variation in leaf form and other characters of Solanum tuberosum group Andigena. European Potato Journal 11: 277-280.

Glendinning, D.R. 1969. The performance of progenies obtained by crossing Groups Andigena and Tuberosum of Solanum tuberosum. European Potato Journal 12: 13-19.

Glendinning, D.R. 1975a. Neo-Tuberosum: New potato breeding material. 1. The origin, composition, and development of the Tuberosum and Neo-Tuberosum gene pools. Potato Research 18: 256-261.

Glendinning, D.R. 1975b. Neo-Tuberosum: New potato breeding material. 2. A comparison of Neo-Tuberosum with unselected Andigena and with Tuberosum. Potato Research 18: 343-350. 
Grun, P. 1990. The evolution of cultivated potatoes. Economic Botany 44 (Suppl. 3): 39-55.

Grun, P., C. Ochoa, and D. Capage. 1977. Evolution of cytoplasmic factors in tetraploid cultivated potatoes (Solanaceae). American Journal of Botany 64: 412-420.

Hamilton, J.P., C.N. Hansey, B.R. Whitty, K. Stoffel, A.N. Massa, A. Van Deynze, W.S. De Jong, D.S. Douches, and C.R. Buell. 2011. Single nucleotide polymorphism discovery in elite north American potato germplasm. BMC Genomics 12: 302.

Hawkes, J.G. 1990. The potato - evolution, biodiversity and genetic resources. London: Belhaven Press.

Hermundstad, S.A. 1986. Haploid-wild species hybrids in potato breeding, genetics, and germplasm enhancement. Ph.D. Thesis, University of Wisconsin-Madison.

Hirsch, C.N., C.D. Hirsch, K. Felcher, J. Coombs, D. Zarka, A. Van Deynze, W. De Jong, R.E. Veilleux, S. Jansky, P. Bethke, D.S. Douches, and C.R. Buell. 2013. Retrospective view of North American potato (Solanum tuberosum L.) breeding in the 20th and 21 st centuries. G3 Genes Genomes Genetics 3: 1003-1013.

Hosaka, K., and R.E. Hanneman Jr. 1988. The origin of the cultivated tetraploid potato based on chloroplast DNA. Theoretical and Applied Genetics 76: 172-176.

Hosaka, K., and R.E. Hanneman Jr. 1993. Effects of different chloroplast DNA types on tuber yield in the Andean and Chilean tetraploid potatoes-Preliminary report. Science Reports of Faculty of Agriculture, Kobe University 20: 177-181.

Hosaka, K., and R.E. Hanneman Jr. 1998. Genetics of self-compatibility in a self-incompatible wild diploid potato species Solanum chacoense. 2. Localization of an S locus inhibitor (Sli) gene on the potato genome using DNA markers. Euphytica 103: 265-271.

Hosaka, K., and R. Sanetomo. 2012. Development of a rapid identification method for potato cytoplasm and its use for evaluating Japanese collections. Theoretical and Applied Genetics 125: 1237-1251.

Hosaka, K., R. Sanetomo, S. Tamiya, K. Asano, and E. Shimosaka. 2018. Creation of long-day adapted tetraploid potatoes from crosses of Solanum tarijense females with Andigena males. Euphyitica 214: 191

Huarte, M.A., and R.L. Plaisted. 1984. Selection for Tuberosum likeness in the vines and in the tubers in a population of Neotuberosum. American Potato Journal 61: 461-473.

Huijsman, C.A. 1955. Breeding for resistance to the potato root eelworm. II. Data on the inheritance in andigenum-tuberosum crosses obtained in 1954. Euphytica 4: 133-140.

Igarashi, T., M. Tsuyama, K. Ogawa, E. Koizumi, R. Sanetomo, and K. Hosaka. 2019. Evaluation of Japanese potatoes using single nucleotide polymorphisms (SNPs). Molecular Breeding 39: 9.

Iketani, S., K. Senda, M. Iritani, T. Itoh, K. Sekiguchi, M. Ohnami, and R. Fujita. 2015. Breeding of a new table potato variety "Saya-akane" with high resistance to Phytophthora infestans and high quality. Breeding Research 17: 25-34.

Jansky, S., and A. Hamernik. 2015. Rapid cycling of potato tuber generations by overcoming dormancy. American Journal of Potato Research 92: 148-152.

Kawano, K. 2003. Thirty years of cassava breeding for productivity-biological and social factors for success. Crop Science 43: 1325-1335.

Kloosterman, B., J.A. Abelenda, M.M.C. Gomez, M. Oortwijn, J.M. de Boer, K. Kowitwanich, B.M. Horvath, H.J. van Eck, C. Smaczniak, S. Prat, R.G.F. Visser, and C.W.B. Bachem. 2013. Naturally occurring allele diversity allows potato cultivation in northern latitudes. Nature 495: 246-250.

Leonards-Schippers, C., W. Geffers, F. Salamini, and C. Gebhardt. 1992. The $R 1$ gene conferring race-specific resistance to Phytophthora infestans in potato is located on potato chromosome V. Molecular and General Genetics 233: 278-283.

Leonards-Schippers, C., W. Gieffers, R. Schäfer-Pregl, E. Ritter, S.J. Knapp, F. Salamini, and C. Gebhardt. 1994. Quantitative resistance to Phytophthora infestans in potato: A case study for QTL mapping in an allogamous plant species. Genetics 137: 67-77.

Lössl, A., M. Götz, A. Braun, and G. Wenzel. 2000. Molecular markers for cytoplasm in potato: Male sterility and contribution of different plastid-mitochondrial configurations to starch production. Euphytica 116: 221-230.

Maris, B. 1989. Analysis of an incomplete diallel cross among three ssp. tuberosum varieties and seven long-day adapted ssp. andigena clones of the potato (Solanum tuberosum L.). Euphytica 41: 163 182.

Massa, A.N., N.C. Manrique-Carpintero, J.J. Coombs, D.G. Zarka, A.E. Boone, W.W. Kirk, C.A. Hackett, G.J. Bryan, and D.S. Douches. 2015. Genetic linkage mapping of economically important traits in cultivated tetraploid potato (Solanum tuberosum L.). G3 Genes Genomes Genetics 5: 2357-2364.

Mendoza, H.A., and F.L. Haynes. 1974. Genetic relationship among potato cultivars grown in the United States. HortScience 9: 328-330.

Mihovilovich, E., R. Sanetomo, K. Hosaka, B. Ordoñez, M. Aponte, and M. Bonierbale. 2015. Cytoplasmic diversity in potato breeding: Case study from the International Potato Center. Molecular Breeding 35: 13.

Mori, M., S. Tsuda, N. Mukojima, A. Kobayashi, C. Matsuura-Endo, A. Ohara-Takada, and I.S.M. Zaidul. 2007. Breeding of potato cyst nematode resistant varieties in Japan. In Potato production and innovative technologies, ed. A.J. Haverkort and B.V. Anisimov, 328339. Wageningen: Wageningen Academic Publishers.

Mori, K., Y. Sakamoto, N. Mukojima, S. Tamiya, T. Nakao, T. Ishii, and K. Hosaka. 2011. Development of a multiplex PCR method for simultaneous detection of diagnostic DNA markers of five disease and pest resistance genes in potato. Euphytica 180: 347-355.

Mori, K., N. Mukojima, T. Nakao, S. Tamiya, Y. Sakamoto, N. Sohbaru, K. Hayashi, H. Watanuki, K. Nara, K. Yamazaki, T. Ishii, and K. Hosaka. 2012. Germplasm release: Saikai 35, a male and female fertile breeding line carrying Solanum phureja-derived cytoplasm and potato cyst nematode resistance (HI) and Potato virus $Y$ resistance $\left(R y_{c h c}\right)$ genes. American Journal of Potato Research 89: 63 72 .

Munoz, F.J., and R.L. Plaisted. 1981. Yield and combining abilities in Andigena potatoes after six cycles of recurrent phenotypic selection for adaptation to long day conditions. American Potato Journal 58: 469-479.

Munoz, F.J., R.L. Plaisted, and H.D. Thurston. 1975. Resistance to potato virus Y in Solanum tuberosum ssp. andigena. American Potato Journal 52: 107-115.

Ohbayashi, K., N. Nakata, M. Chaya, and K. Komura. 2010. Development of a detection method of resistance to potato disease and pest using DNA markers. 1. Detection methods of resistance to potato virus $X$, potato cyst nematode and late blight. Bulletin of Nagasaki Agricultural and Forestry Technical Development Center 1: 1-26.

Peterson, B.A., S.H. Holf, F.P.E. Laimbeer, A.G. Doulis, J. Coombs, D.S. Douches, M.A. Hardigan, C.R. Buell, and R.E. Veilleux. 2016. Selffertility in a cultivated diploid potato population examined with the Infinium 8303 potato single-nucleotide polymorphism array. The Plant Genome 9: 3.

Pineda, O., M.W. Bonierbale, R.L. Plaisted, B.B. Brodie, and S.D. Tanksley. 1993. Identification of RFLP markers linked to the $H 1$ gene conferring resistance to the potato cyst nematode Globodera rostochiensis. Genome 36: 152-156.

Plaisted, R.L., and R.W. Hoopes. 1989. The past record and future prospects for the use of exotic potato germplasm. American Potato Journal 66: 603-627.

Plaisted, R.L., D.E. Halseth, B.B. Brodie, S.A. Slack, J.B. Sieczka, B.J. Christ, K.M. Paddock, and M.W. Peck. 1998. Pike: A full season scab and golden nematode resistant chipstock variety. American Journal of Potato Research 75: 117-120. 
Provan, J., W. Powell, H. Dewar, G. Bryan, G.C. Machray, and R. Waugh. 1999. An extreme cytoplasmic bottleneck in the modern European cultivated potato (Solanum tuberosum) is not reflected in decreased levels of nuclear diversity. Proceedings of the Royal Society B 266: 633-639.

Rasco, E.T., Jr., R.L. Plaisted, and E.E. Ewing. 1980. Photoperiod response and earliness of $S$. tuberosum ssp. andigena after six cycles of recurrent selection for adaptation to long days. American Potato Journal 57: 435-447.

Ritter, E., T. Debener, A. Barone, F. Salamini, and C. Gebhardt. 1991. RFLP mapping on potato chromosomes of two genes controlling extreme resistance to potato virus X (PVX). Molecular and General Genetics 227: 81-85.

Ross, H. 1986. Potato breeding-problems and perspectives. Berlin: Verlag Paul Parey.

Ross, H., and C.A. Huijsmann. 1969. Über die Resistenz von Solanum (Tuberarium)-Arten gegen europäische Rassen des Kartofielnematoden (Heterodera rostochiensis Woll.). Theoretical and Applied Genetics 39: 113-122.

Sakaguchi, S., Y. Umemura, Y. Okuyama, Y. Irikura, N. Takase, K. Tabata, T. Nagata, and H. Oka. 1976. A new processing potato variety "Toyoshiro". Research Bulletin of the Hokkaido National Agricultural Experiment Station 116: 95-109.

Salaman, R.N. 1946. The early European potato: Its character and place of origin. Botanical Journal of the Linnean Society 53: 1-27.
Simmonds, N.W. 1964. Studies of the tetraploid potatoes. I. The variability of the Andigena Group. Botanical Journal of the Linnean Society 58: 461-474.

Simmonds, N.W. 1966. Studies of the tetraploid potatoes. III. Progress in the experimental re-creation of the Tuberosum Group. Botanical Journal of the Linnean Society 59: 279-288.

Song, Y.-S., and A. Schwarzfischer. 2008. Development of STS markers for selection of extreme resistance $\left(R y_{\text {sto }}\right)$ to PVY and maternal pedigree analysis of extremely resistant cultivars. American Journal of Potato Research 85: 159-170.

Sukhotu, T., O. Kamijima, and K. Hosaka. 2005. Genetic diversity of the Andean tetraploid cultivated potato (Solanum tuberosum L. subsp. andigena Hawkes) evaluated by chloroplast and nuclear DNA markers. Genome 48: 55-64.

Tarn, T.R., and G.C.C. Tai. 1977. Heterosis and variation of yield components in $F_{1}$ hybrids between Group Tuberosum and Group Andigena potatoes. Crop Science 17: 517-521.

Uitdewilligen, J.G.A.M.L., A.-M.A. Wolters, B.B. D'hoop, T.J.A. Borm, R.G.F. Visser, and H.J. van Eck. 2013. A next-generation sequencing method for genotyping-by-sequencing of highly heterozygous autotetraploid potato. PLoS One 8: e62355.

Vos, P.G., J.G.A.M.L. Uitdewilligen, R.E. Voorrips, R.G.F. Visser, and H.J. van Eck. 2015. Development and analysis of a 20K SNP array for potato (Solanum tuberosum): An insight into the breeding history. Theoretical and Applied Genetics 128: 2387-2401. 